\title{
Angiosarcoma epitelioide de vesícula. Presentación de un caso y revisión bibliográfica
}

\section{Epithelioid angiosarcoma of the gallbladder. Case report and review of the literature}

\author{
P. Sánchez Acedo, J. Herrera Cabezón, A. Tarifa Castilla, C. Zazpe Ripa, J.M. Lera Tricas
}

\section{RESUMEN}

El angiosarcoma de vesícula es una patología poco frecuente pero con una alta morbi-mortalidad del que sólo hay 10 referencias en la literatura internacional.

Presentamos un caso tratado en nuestro centro y realizamos una revisión de los casos publicados desde 1956.

Paciente varón de 81 años con dolor abdominal, astenia y disnea. Analíticamente anemia y leucocitosis. En la exploración destacaba un abdomen distendido, doloroso en hipocondrio derecho, con defensa. Se solicitó ecografía abdominal y un TC con diagnóstico de colecistitis aguda y se ingresó para tratamiento antibiótico. El paciente no evolucionó favorablemente y se intervino de urgencia hallando un hemoperitoneo y una vesícula de aspecto tumoral que no se pudo extirpar en su totalidad. Falleció a los 20 días de la intervención. El informe de anatomía patológica fue compatible con angiosarcoma epiteloide de vesícula biliar.

El angiosarcoma de vesícula es una neoplasia de mal pronóstico, cuya presentación clínica puede confundirse con la colecistitis aguda. Conseguir mejorar el pronóstico de esta enfermedad pasa por realizar un diagnóstico y tratamiento quirúrgico precoces.

Palabras clave. Angiosarcoma epitelioide. Neoplasia. Vesícula biliar.

\begin{abstract}
Angiosarcoma of the gallbladder is an infrequent pathology but has a high morbidity and mortality. There are only 10 references in the international literature.

We present a case treated in our center and we review the cases published since 1956 .

An 81 year-old male patient with abdominal pain, asthenia and dyspnea. Analytically anemia and leukocytosis. Exploration found a distended abdomen, right hypochondrium pain, with defense. Abdominal echography and a CT were requested with a diagnosis of acute cholecystitis and he was admitted for antibiotic treatment. The patient did not evolve favorably and was subjected to emergency surgery, which found a haemoperitoneum and a gallbladder with a tumoral appearance that could not be totally extirpated. He died 20 days after the operation. The report from pathological anatomy was compatible with epithelioid angiosarcoma of the biliary gallbladder.

Gallbladder angiosarcoma is a neoplasia with a bad prognosis, whose clinical presentation can be mistaken for acute cholecystitis. Improving the prognosis of this disease involves carrying out early diagnosis and surgical treatment.
\end{abstract}

Keywords. Epithelioid angiosarcoma. Neoplasia. Biliary gallbladder.
Servicio de Cirugía General y del Aparato Digestivo. Complejo Hospitalario de Navarra. Pamplona

Recepción: 18 de noviembre de 2014

Aceptación provisional: 2 de marzo de 2015

Aceptación definitiva: 3 de marzo de 2015

\section{Correspondencia:}

Pablo Sánchez Acedo

Servicio de Cirugía general y del Aparato digestivo Complejo Hospitalario de Navarra 31008 Pamplona

E-mail: pablosanchez_84@hotmail.com 


\section{INTRODUCCIÓN}

El término angiosarcoma hace referencia a un amplio rango de neoplasias malignas que afectan al endotelio vascular de gran variedad de órganos y tejidos. Son neoplasias poco frecuentes que se caracterizan por un crecimiento rápidamente proliferativo e infiltrante de células anaplásicas derivadas del endotelio vascular. Recientemente se ha descrito la variante epitelioide, más agresiva, con una supervivencia inferior a los 2-3 años.

El angiosarcoma de vesícula es una rara neoplasia de la que solo hay 10 casos recogidos en la literatura médica desde $1956^{1-10}$. Presentamos un nuevo caso tratado en el Complejo Hospitalario de Navarra y realizamos una revisión de la bibliografía disponible.

\section{CASO CLÍNICO}

Paciente varón de 81 años de edad, con antecedentes de hipertensión arterial, fibrilación auricular (por la que se encontraba en tratamiento anticoagulante) y adenocarcinoma de próstata por el que había sido intervenido y se encontraba libre de enfermedad.

Acudió al servicio de Urgencias por un episodio de dolor precordial irradiado a hipocondrio derecho de varias horas de evolución, acompañado de disnea de mínimos esfuerzos. En el momento de la valoración la sintomatología había disminuido. Inicialmente se diagnosticó como un posible síndrome coronario agudo por lo que se inició tratamiento y se solicitó una analítica con enzimas de daño miocárdico y un electrocardiograma que no presentaban anomalías. El paciente refería astenia y molestias en hipocondrio derecho de 7 días de evolución, pero en el momento de la valoración no presentaba dolor y la exploración física era anodina por lo que tras ser valorado por el servicio de Cardiología se dio de alta.

Seis días después el paciente acudió al servicio de Urgencias por persistencia del dolor en hipocondrio derecho acompañado de naúseas e hiporexia. No presentaba fiebre ni otra clínica acompañante. A la exploración destacaba un abdomen distendido, doloroso a la palpación en hipocondrio y flanco derecho con defensa pero con signos de Blumberg y Murphy negativos. Se realizó una analítica en la que se observaba una hemoglobina de $11 \mathrm{~g} / \mathrm{dl}$, hematocrito de $32,6 \%$, leucocitos 14.000 con $74 \%$ de neutrófilos, INR 2,95 y fibrinógeno $816 \mathrm{mg} / \mathrm{dl}$.

Se solicitó una ecografía abdominal en la que se observó una vesícula con paredes mal definidas y colelitiasis, además de una imagen hipoecoica compatible con líquido libre perivesicular que se interpretó como colecistitis aguda perforada y se decidió completar el estudio con una tomografía computarizada (TC). En la TC se confirmó la presencia de una vesícula con paredes mal definidas junto a una colección de líquido de alta densidad de $7 \times 3 \mathrm{~cm}$ que alteraba la grasa circundante compatible con colecistitis aguda perforada (Fig. 1).

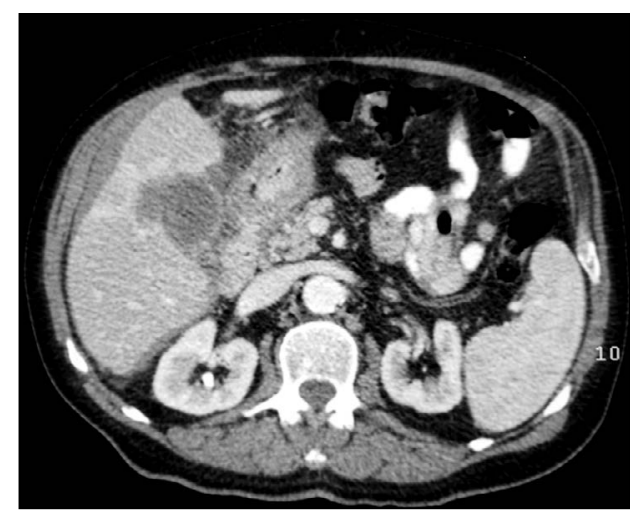

Figura 1. Colecistitis aguda perforada.

El paciente se encontraba estable y no presentaba signos de irritación peritoneal en la exploración por lo que se decidió el ingreso en la planta de Cirugía para tratamiento conservador. Se instauró tratamiento analgésico y antibiótico (Piperacilina-Tazobactan). A las 48 horas del ingreso el paciente continuaba con molestias en hipocondrio derecho y en los análisis de control persistía la leucocitosis por lo que se decidió realizar una TC de control (Fig. 2). En la prueba de imagen se confirmó la mala evolución del proceso con aumento del líquido libre intraabdominal. Con estos hallazgos se realizó una intervención quirúrgica urgente.

Fue intervenido mediante una incisión subcostal derecha por la que se accedió a la cavidad peritoneal. Se localizó un plastrón de epiplón que cubría la vesícula, de aspecto tumoral, con sufusión hemorrágica en espacio subfrénico y perirrenal, así como un hemoperitoneo de 2,7 litros sin apreciarse punto de hemorragia. Se realizó una colecistectomía subtotal por invasión tumoral del parénquima hepático y se extirpó la mayor parte del epiplón quedando residuo tumoral macroscópico. 


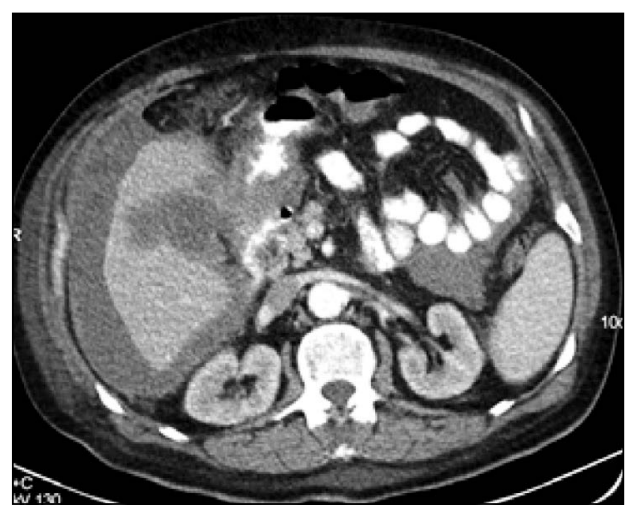

Figura 2. TC donde se observa aumento del líquido intraabdominal.

La evolución postoperatoria fue favorable y el paciente fue dado de alta al $6^{\circ}$ día postoperatorio.

A la semana del alta, el paciente reingresó por dolor abdominal en hipocondrio derecho asociado a astenia y anorexia. En los análisis se observó una hemoglobina de 7,5 g/dL, hematocrito de $22,7 \%$ y 32.500 leucocitos con desviación izquierda. El paciente presentaba mal estado general, con dolor en mesogastrio e hipocondrio derecho. Se realizó una ecografía abdominal en la que se apreció una colección de $4 \times 4 \mathrm{~cm}$ en el lecho quirúrgico.

Se estableció tratamiento sintomático pero el paciente presentó un deterioro progresivo durante los días siguientes marcado por disnea, dolor abdominal en aumento, melenas y una anemización progresiva asociada a hipotensión a pesar de transfusiones por lo que finalmente falleció a los 20 días de ser intervenido.

Se realizó el estudio de las muestras remitidas a anatomía patológica. En ellas se identificaron áreas con un infiltrado en células entremezclado con focos hemorrágicos y zonas en donde las células se disponen formando canales vasculares (Fig. 3).

Se completó el estudio con un análisis inmunohistoquímico en el que destacaba la queratina 7 positivo intenso, D2.40, CD-31 y vimentina intensamente positivo. Todo ello compatible con el diagnóstico de angiosarcoma epitelioide primario de vesícula biliar.

\section{DISCUSIÓN}

El angiosarcoma de vesícula, como se puede apreciar en el presente caso, es una patología muy agresiva, con un pronósti-

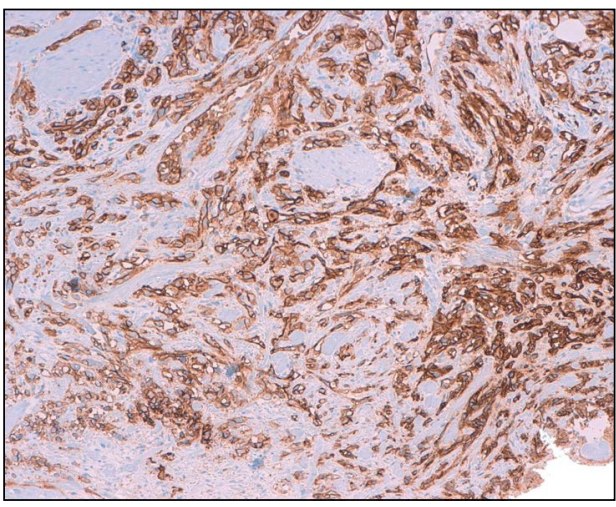

Figura 3. Infiltrado celular con células en canales vasculares.

co ominoso y de difícil diagnóstico. Revisando la literatura disponible, las primeras descripciones de este tumor datan de $1956^{1}$ y $1972^{2}$, pero los resultados de estos artículos no se encuentran disponibles. Del resto de los casos descritos en la literatura desde $1982^{3-10}$ se obtienen los datos que se recogen en la tabla 1.

Es un tumor que se desarrolla en pacientes adultos, con un rango de edad entre los 54 y los 87 años. Afecta predominantemente a varones, con una proporción de 2 a 1 , al igual que sucede en el resto de los angiosarcomas ${ }^{11}$.

Existe una gran dificultad a la hora de diagnosticar y tratar esta patología ya que tiene una incidencia muy baja y una clínica inespecífica por lo que en la mayoría de los casos el diagnóstico se produce con los resultados de la anatomía patológica.

La clínica más frecuente descrita consiste en dolor en hipocondrio derecho, acompañado o no de una masa palpable, lo que hace que generalmente se oriente el caso como una colecistitis aguda.

En dos de los $\operatorname{casos}^{6,10}$ los pacientes referían astenia y melenas durante las semanas previas. Nuestro caso también debutó con un episodio de disnea con astenia y presentó melenas en el postoperatorio. Hay que recordar que se trata de un tumor que afecta a los vasos con un importante riesgo de sangrado por lo que generalmente se asocia a hemoperitoneo y el shock 
Tabla 1. Datos sobre 1956 a 2010 de trabajos consultados

\begin{tabular}{lcccccc}
\hline \multicolumn{1}{c}{ Autor } & Año & Edad & Sexo & Clínica & Tratamiento & Supervivencia \\
\hline Belelli & 1956 & & & - & - & \\
\hline Vaittinen, E & 1972 & & & & & \\
\hline Rosansky & 1982 & 59 & varón & Dolor HCD + Masa palpable & Médico & 28 días \\
\hline Kawai & 1989 & 63 & varón & Dolor HCD + Masa palpable & Colecistectomía & 4 meses \\
\hline Kumar & 1989 & 56 & varón & Masa palpable & Colecistectomía & 5 meses \\
\hline Hittmair & 1991 & 87 & mujer & Astenia + Melenas & Colecistectomía & 4 días \\
\hline White & 1994 & 81 & mujer & Dolor HCD + Masa palpable & Colecistectomía & 14 días \\
\hline Kumar & 1994 & 54 & varón & Masa palpable & Colecistectomía & 5 años \\
\hline Odashiro & 2005 & 62 & mujer & Dolor HCD & Colecistectomía & 2 días \\
\hline Costantini & 2005 & 57 & varón & Astenia + Melenas + Dolor HCD & Colecistectomía & $>5$ meses \\
\hline Sánchez & 2010 & 81 & varón & Astenia + Dolor HCD & Colecistectomía & 20 días \\
\hline
\end{tabular}

hemorrágico es uno de las causas de fallecimiento de estos pacientes.

La mayor parte de los pacientes fueron sometidos a tomografía computarizada y/o ecografía antes de ser intervenidos; estas pruebas son sensibles para detectar el tumor, pero las imágenes no son lo suficientemente específicas como para diferenciarlo de una colecistitis. No hay resultados en el uso de otras pruebas como la resonancia magnética.

El tratamiento de la mayoría de los $\operatorname{casos}^{4-10}$ fue la colecistectomía. En el tratamiento de los angiosarcomas de partes blandas, la cirugía radical junto a la radioterapia es el tratamiento de elección en tumores no diseminados ${ }^{11}$ pero en el angiosarcoma de vesícula no se ha podido aplicar ya que la enfermedad se encuentra muy evolucionada en el momento del diagnóstico. La quimioterapia preoperatoria se reserva para los casos de angiosarcomas diseminados, pero tampoco se ha podido aplicar a la vesícula porque el diagnóstico es siempre postoperatorio.

La supervivencia postoperatoria de este tumor oscila entre los 2 días $^{9}$ y 5 años $^{8}$.

Uno de los casos más recientes ${ }^{10}$ presenta una supervivencia superior a 5 me- ses libre de enfermedad. A este paciente se le realizó una colecistectomía de urgencia y tras obtener los resultados de la anatomía patológica se reintervino realizando una resección del segmento IV hepático.

El único caso que presenta una supervivencia libre de enfermedad superior a los 5 años $^{8}$ no concuerda con el resto y algunos autores sugieren que se trate en realidad de un carcinoma escamoso de vesícula con cambios en la proliferación capilar.

En conclusión, ante la aparición anemia, melenas o hemoperitoneo asociados a una colecistitis evolucionada con masa o plastrón inflamatorio hay que plantearse la posibilidad de origen neoplásico del cuadro e incluir el angiosarcoma en el diagnóstico diferencial.

En los pacientes con tumores localizados y estado general aceptable, la extirpación radical sin residuo tumoral macroscópico, es la opción que probablemente obtenga mayor supervivencia y un mejor control de los síntomas.

Al tratarse de una patología poco frecuente es necesario comunicar los casos diagnosticados para contribuir a un mayor conocimiento de la patología y poder mejorar pronóstico de estos pacientes. 


\section{BIBLIOGRAFÍA}

1. Belelli E. Case of primary angiosarcoma of the gallbladder. Ann Ital Chir 1956; 33: 11011114.

2. VaitTinen E. Sarcoma of the gall-bladder. Ann Chir Gynaecol Fenn 1972; 61: 185-189.

3. Rosansky SJ, Mullens CC. The hepatorenal syndrome associated with metastatic angiosarcoma of the gallbladder. Ann Intern Med 1982; 96: 191-192.

4. Kawai T, Hirose Y, Ainota T, Miyazaki Y, Yamamoto $\mathrm{Y}$, IKEDA $\mathrm{M}$ et al. A case of hemangiosarcoma of the gallbladder. Nihon Shokakibyo Gakkai Zasshi Jpn. J Gastro-Enterol 1989; 86: 26112616.

5. Kumar A, Lal BK, Singh MK, Kapur BM. Angiosarcoma of the gallbladder. Am J Gastroenterol 1989; 84: 1431-1433.

6. Hittmair A, Sandbichler P, Tötsch M, Mikuz G, ScHмID KW. Primary angiosarcoma of the gall- bladder. Case report with review of the literature. Pathol 1991; 12: 279-281.

7. White J, Chan YF. Epithelioid angiosarcoma of the gallbladder. Histopathology 1994; 24: 269-271.

8. Kumar A, Singh MK, Kapur BM. Synchronous double malignant tumors of the gall bladder. A case-report of squamous cell carcinoma with an angiosarcoma. Eur J Surg Oncol J Eur Soc Surg Oncol Br Assoc Surg Oncol 1994; 20: 63-67.

9. Odashiro an, Pereira Pr, Odashiro MijJ LN, NGUYEN GK. Angiosarcoma of the gallbladder: case report and review of the literature. Can J Gastroenterol J Can Gastroenterol 2005; 19: 257-259.

10. Costantini R, Di Bartolomeo N, Francomano F, Angelucci D, InNocenti P. Epithelioid angiosarcoma of the gallbladder: case report. J Gastrointest Surg Off J Soc Surg Aliment 2005 Tract 9, 822-825. 